\title{
Detecting malaria sporozoites in live, field-collected mosquitoes
}

\begin{abstract}
A. Habluetzel ${ }^{1,2}$, L. Merzagora $^{3}$, L. Jenni ${ }^{4}$, B. Betschart ${ }^{4}$, G. Rotigliano ${ }^{5}$ and F. Esposito ${ }^{1}{ }^{1}$ Department of Molecular, Cell, and Animal Biology, University of Camerino, Via Camerini 2, 62032 Camerino (MC), Italy; ${ }^{2}$ World Health Organization Collaborating Centre for Malaria Epidemiology, c/o Institute of Parasitology, 'La Sapienza' University, Rome, Italy; ' Institute of Parasitology, 'La Sapienza' University, Rome, Italy; ${ }^{4}$ Swiss Tropical Institute, CH-4002 Basel, Switzerland; ${ }^{5}$ Italian Ministry for Foreign Affairs, Directorate General for Co-operation in Development, and Institute of Tropical Diseases (3rd chair), 'La Sapienza' University, Rome, Italy; and Centre de Lutte contre le Paludisme, Ouagadougou, Burkina Faso
\end{abstract}

\begin{abstract}
A method is described for identifying malaria-infected mosquitoes, without killing them or hampering their fitness. Individual mosquitoes were induced to salivate on coverslips, and sporozoites, deposited on the glass surface, were visualized by Giemsa staining. Of 21 mosquitoes found to contain sporozoites by salivary gland dissection, 13 had delivered sporozoites on coverslips. A positive correlation was found between the amount of saliva expelled and cicction of sporozoites, indicating that the sensitivity of the method may be increased by improving the probing behaviour of the mosquitoes. The procedure described may be suitable for selecting infected mosquitoes which are able to eject sporozoites during probing. Being applicable to wild Anopheles and to large numbers of mosquitoes, the method lends itself for use in field studies on malaria.
\end{abstract}

\section{Introduction}

An easy method for assessing whether a single mosquito harbours Plasmodium sporozoites in its salivary glands, and whether it may eject these sporozoites dur ing probing, could be useful for certain studies in malaria research.

Classical methods for identifying malaria sporozoite infection in mosquitoes rely either upon microscopical examination of dissected salivary glands, or upon immunological identification of circumsporozoite (CS) protein in mosquito extracts (Collins et al., 1984).

In trypanosomiasis research, an easy and rapid method for identifying trypanosomes in live tsetse flics was published by BURTT in 1946 and is now widely used. The method exploits the behaviour of Glossina in responding with salivation when stimulated by a warm surface. In practice, flies are offered a lukewarm glass slide and allowed to deposit saliva for some minutes. The slide is then examined microscopically for the presence of trypanosomes.

Here we describe a modified salivation method, which enables Plasmodium sporozoite infections to be detected in live, field-collected Anopheles, and propose possible applications in malaria research.

\section{Material and Methods}

Anopheles gambiae s.l. mosquitoes were collected in Noungou, a village near Ougadougou (Burkina Faso), in which $P$. falciparum sporozoite rates were about $10 \%$ during the study period. The hand-caught females were maintained for $10-12 \mathrm{~d}$ in an insectary on $1 \%$ sugar, to allow the sporogonic cycle to be completed and a higher percentage of salivary gland-infected specimens to be obtained. For the salivation experiments, mosquitoesplaced individually in paper cups covered with gauze-were stimulated to feed by gently breathing into the cup and by placing the forearm over it. As soon as a female showed exploratory behaviour, searching for a host, a coverslip, warmed by a finger, was held on the gauze. Mosquitoes were allowed to probe and salivate on the glass surface for 5 to $10 \mathrm{~min}$, being restimulated at intervals. Their salivary glands were then dissected and examined microscopically for sporozoites. Coverslips from mosquitoes with sporozoites were fixed with methanol, stained with Giemsa's stain and mounted on slides with Canada balsam. The coverslips were examined for the presence of saliva spots at $100 \times$ magnification, and sporozoites were counted at $600 \times$ magnification. The amount of expelled saliva was arbitrarily assessed, considering as 1 saliva unit a single saliva deposit or-when saliva had been distributed over an extended area-one- fifth of the microscopical ficld at $600 \times$ magnification.

\section{Results}

Screening for Plasmodium sporozoite infection was performed on about 200 field-collected Anopheles females, by stimulating them individually to salivate on coverslips. At dissection, 21 of these mosquitoes were found to harbour sporozoites in their salivary glands. Of these, 13 had expelled saliva containing sporozoites. The amount of saliva deposited per coverslip varied widely. A positive correlation (Spearman's rank correlation coefficient $r_{\mathrm{s}}=0.72, P<0.001$ ) was found between the number of sporozoite-positive saliva units per coverslip and the total number of saliva units per coverslip (Figure). Acc-

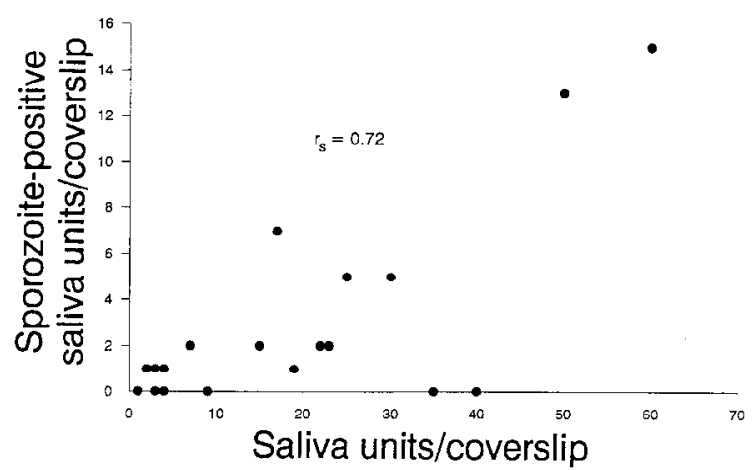

Figure. Correlation between sporozoite-positive saliva units per coverslip and total number of saliva units per coverslip. The value of the Spearman's rank correlation coefficient, $r_{\mathrm{s}}=0.72$, is significantly different from $0(P<0-001)$

ordingly, sporozoites were found on 9 of the 11 coverslips on which mosquitoes deposited a number of saliva units higher than or equal to the median (median $=15$, geometric mean $=10 \cdot 25$ ), while only 4 of the 10 coverslips with fewer than 15 saliva units contained sporozoites. On average, about one-fifth of the saliva units on sporozoite-containing coverslips were positive (geometric mean of the ratio positive/total saliva units $=0 \cdot 19$, standard deviation $[\mathrm{SD}]=0 \cdot 13$, range $0 \cdot 05-0 \cdot 5$ ).

As shown in the Table, usually 1 to 3 sporozoites were found in sporozoite-positive saliva units (mode $=1$; geometric mean $=2 \cdot 3, \mathrm{SD}=7 \cdot 5$ ) with the exception of one coverslip, on which up to 30 sporozoites were noted in one saliva unit. Considering the coverslips as a whole, a geometric mean of 4.4 sporozoites per positive coverslip was recorded $(S \mathrm{SD}=43 \cdot 3$; range $=1-166)$. 
Table. Number of sporozoites ejected by individual mosquitoes

\begin{tabular}{cc}
\hline \multicolumn{2}{c}{ No. of sporozoites } \\
in each positive saliva unit & per coverslip \\
\hline 1 & 1 \\
1 & 1 \\
1,1 & 2 \\
1,1 & 2 \\
1,1 & 2 \\
1,1 & 2 \\
6 & 6 \\
$1,1,1,1,2$ & 6 \\
$1,1,1,2,3$ & 8 \\
$1,1,1,1,2,2,2$ & 10 \\
$1,1,1 \ldots 3,3,4^{\mathrm{a}}$ & 29 \\
$2,5,6 \ldots 18,26,30^{\mathrm{b}}$ & 166 \\
\hline
\end{tabular}

${ }^{\mathrm{a}} 15$ saliva units were deposited.

${ }^{\mathrm{b}} 13$ saliva units were deposited.

\section{Discussion}

In trypanosomiasis research a salivation method, based on stimulation by warmth, is widely used to identify infected flies (BURTT, 1946). This method, slightly modified, proved to be applicable for detecting Plasmodium sporozoite infection in live, field-collected $A n$. gambiae s.l. Sporozoite-containing saliva deposits were found on about two-thirds (13/21) of the coverslips on which mosquitoes known to be positive by dissection had been probing. Although this proportion of positive coverslips may appear relatively low, taking into account the positive correlation between sporozoite-containing saliva units and the amount of saliva deposited, it could probably be augmented by improving the probing behaviour of the mosquitoes.

Since the $1930 \mathrm{~s}$, several attempts have been made to estimate the number of sporozoites a mosquito injects during its bloodmeal (reviewed by ROSENBERG et al., 1990). This topic has enjoyed renewed interest in recent years in connection with research aimed at developing an anti-sporozoite malaria vaccine (DAvIS et al., 1989a, 1989b; RICKMAN et al., 1990; HERRINGTON et al., 1990). Obviously, the salivation method described here, in which a glass surface was used as probing substrate, was not designed to determine the size of parasite inocula. Nevertheless, the results obtained $(4.4$ sporozoites ejected on average per mosquito, range 1-166) were of the same order of magnitude as estimates of sporozoite inocula derived from different experimental salivation or feeding systems: examining wild Anopheles, BEIER et al. (1991) reported a value 3.8 sporozoite (range 1-34); studying experimentally infected mosquitoes, RoSENBERG et al. (1990) and PONNUDURAI et al. (1991) reported values of 15 (range $0-978$ ) and 8 sporozoites (range 0 522 ), respectively. Interestingly, in the studies referred to, as well as in ours, the upper limit of the range of sporozoites expelled was many times higher than the average value, indicating that single infected bites may deliver a sporozoite load much heavier than that injected by the majority of the mosquito bites.

Our finding that 2 infected mosquitoes, although salivating abundantly (more than twice the median number of saliva units), did not deposit a single sporozoite on the coverslip, was also consistent with previous observations by ROSENBERG et al. (1990)-who observed that 9 of the 46 mosquitoes which delivered more than the median amount of saliva did not expel any sporozoites-and PONNUDURAI et al. (1991), who, using an experimental design closely matching the natural fecding situation, rcported that 9 of 34 mosquitoes failed to inject sporozoites. An explanation for these observations may be found in the architecture of the salivary gland, due to which only sporozoites which have penetrated the distal acinar cells can gain access to the duct lumen (STERLING et al., 1973; PonNuduraI et al., 1991). Mosquitoes which salivate abundantly but do not expel sporozoites might represent specimens with infections limited to the proximal portions of the salivary glands. Whatever the reason may be, experimental data agree that some sporozoite-harbouring mosquitoes do not eject sporozoites. If it were demonstrated that the inability of infected mosquitoes to eject sporozoites is an inherent property of individuals, the accuracy of entomological inoculation rates, based on the classical sporozoite rate, may have to be questioned.

The results obtained suggest that the method described here may be used for selecting, from a group of malaria-infected mosquitoes, (i) those which are certainly able to eject sporozoites during probing and (ii) those ones which are capable of expelling exceptionally large sporozoite loads. A method with these characteristics might be useful, for example, to select for high or low transmission capacity among laboratory-reared mosquito colonies. An additional feature of the method, which might be useful in field applications, would be the specific identification of the ejected sporozoites. This could be easily done, by processing the slides for immunofluorescence with a species-specific monoclonal antibody (A. Habluetzel and F. Esposito, unpublished data). Recently, a method has been described in which infected Anopheles were induced to salivate on a fructose-soaked nitrocellulose membrane and CS protein on the membrane was revealed by a monoclonal antibody (BILLING SLEY et al., 1991). Since sporozoites shed CS protein during their stay in the salivary glands (PosThumA et al., 1989), a positive reaction in this assay is not conclusive as to whether sporozoites or just CS protein had been deposited with the saliva.

Being applicable to field-collected Anopheles and to large numbers of mosquitoes, the salivation method described here lends itself for use in field studies. An interesting application might be in vaccine efficacy trials: by preliminarily screening wild Anopheles with the salivation method, volunteers could be challenged with a chosen number of vectors ejecting truly natural malaria parasites instead of strains reared in the laboratory.

\section{Acknowledgements}

We sincerely thank the personnel of the Centre de Lutte contre le Paludisme in Ouagadougou, Burkina Faso, for their skilful collahoration, and Tivi Ponnudurai, Ron Rosenberg and Peter Billingsley for their helpful comments on the manuscript This study was supported by the Italian National Research Council (CNR), the Italian Ministry for Foreign Affairs (Directorate General for Co-operation in Development), the International Atomic Energy Agency, the Italian Ministry for University and Scientific Research and the Istituto PasteurCenci Bolognetti Foundation.

\section{References}

Beier, J. C., Onyango, F. K., Koros, J. K., Ramadhan, M. Ogwang, R., Wirtz, R. A., Koech, D. K. \& Roberts, C. R (1991). Quantitation of malaria sporozoites transmitted in vitro during salivation by wild Afrotropical Anopheles. Medical and Veterinary Entomology, 5, 71-79.

Billingsley, P. F., Hodivala, K. J., Winger, L. A. \& Sinden, R. E. (1991). Detection of mature malaria infections in live mosquitoes. Transactions of the Royal Society of Tropical Medicine and Hygiene, 85, 450-453.

Burtt, E. (1946). Salivation by Glossina morsitans on to glass slides: a technique for isolating infected flies. Annals of Tropical Medicine and Parasitology, 40, 141-144.

Collins, F. H., Zavala, F., Graves, P. M., Cochrane, A. H., Gwadz, R. W., Akoh, J. \& Nussenzweig, R. S. (1984). First ficld trial of an immunoradiometric assay for the detection of malaria sporozoites in mosquitoes. American fournal of Tropical Medicine and Hygiene, 33, 538-543.

Davis, I. R., Murphy, J. R., Clyde, D. F., Baqar, S., Cochrane, A. H., Zavala, F. \& Nussenzweig, R. S. (1989a). Estimate of Plasmodium falciparum sporozoite content of Anopheles stephensi used to challenge human volunteers. American Journal of Tropical Medicine and Hygiene, 40, 128130 
Davis, J. R., Murphy, J. R., Baqar, S., Clyde, D. F., Herrington, D. A. \& Levine, M. M. (1989b). Estimate of anti-Plasmodium falciparum sporozoite activity in humans vaccinated with synthetic circumsporozoite protein (NANP) 3 . Transactions of the Royal Society of Tropical Medicine and Hygiene, 83, $748-750$.

Herrington, D. A., Clyde, D. F., Davis, J. R., Baqar, S., Murphy, J. R., Cortese, J. F., Bank, R. S., Nardin, E., DiJohn, D., Nussenzweig, R. S., Nussenzweig, V., Torres, J. R., Murillo, J, Cortesia, M., Stuerchler, D., Hollingdale, M. R. \& Levine, M. M. (1990). Human studies with synthetic peptide sporozoite vaccine (NANP) 3 -TT and immunization with irradiated sporozoites. Bulletin of the World Health $\mathrm{O}_{7}$ ganization, 68, supplement, 33-37.

Ponnudurai, T., Lensen, A. H. W., van Gemert, G. J. A., Bolmer, M. G. \& Meuwissen, J. H. E. Th. (1991). Feeding behaviour and sporozoite ejection by infected Anopheles stephensi. Transactions of the Royal Society of Tropical Medicine and Hygiene, 85, 175-180.

Posthuma, G., Meis, J. F. G. M., Verhave, J. P., Gigengack, S., Hollingdale, M. R., Ponnudurai, T. \& Geuze, H. J
(1989). Immunogold determination of Plasmodium falciparum circumsporozoite protein in Anopheles stephensi salivary gland cell. European fournal of Cell Biology, 49, 66-72.

Rickman, L. S., Jones, T. R., Lond, G. W., Paparello, S., Schneider, I., Paul, C. F., Beaudion, R. L. \& Hoffman, S. L. (1990). Plasmodium falciparum-infected Anopheles stephensi do inconsistently transmit malaria to humans. American fournal of Tropical Medicine and Hygiene, 43, 441-445.

Rosenberg, R., Wirli, R. A., Schneider, I. \& Burge, R. (1990). An estimation of the number of malaria sporozoites ejected by a feeding mosquito. Transactions of the Royal Society of Tropical Medicine and ILygiene, 84, 209-212.

Sterling, C. R., Aikawa, M. \& Vanderberg, J. P. (1973). The passage of Plasmodium berghei sporozoites through the salivary glands of Anopheles stephensi: an electron microscope study. fournal of Parasitology, 59, 593-605.

Received 31 fuly 1991; revised 19 September 1991; accepted for publication 19 September 1991

\section{Announcement}

\section{PRIZES}

\section{UNDERGRADUATE PROJECT PRIZE}

The Royal Society of Tropical Medicine and Hygiene offers an annual prize of $£ 200$ for an account of work carried out in a tropical or developing country by a non-medical student of any nationality. The work will add to the knowledge of human or veterinary health or hygiene in the broadest sense. Particular attention will be directed towards originality and quality in the award of the prize. It is anticipated that the prize will act as a stimulus for the pursuit of excellence in research carried out by undergraduates.

\section{MEDICAL STUDENT ELECTIVE PRIZE}

The Royal Society of Tropical Medicine and Hygiene offers an annual prize of $£ 200$ for an account of work carried out by a British medical student during an elective period spent in a tropical or developing country. In awarding this prize emphasis will be laid on the originality of the work and on its contribution to knowledge or understanding of tropical diseases.

\section{RULES}

1. Two prizes of $£ 200$ may be awarded annually in recognition of outstanding projects which increase knowledge of tropical medicine and hygiene in the broadest sense.

2. Candidates shall be nominated by their head of department, supervisor or Dean, with a supporting statement of up to 500 words.

3. The closing date for receipt of project reports is 31 December. The project should have been done or completed in the previous twelve months.

4. A Committee of three shall choose the prize winners

5. The announcement of the prize winners will be made at the March mecting of the Society.

6. The prizes will be presented by the President of the Society at the Annual General Meeting in June or July.

Please note that the Society cannot provide funds to cover students' elective travel expenses. 